RENAN LUCAS SIENA DEL BEL

RECRUTAMENTO DE ÁRVORES EM UMA FLORESTA DE RESTINGA:

INFLUÊNCIAS DA VIZINHANÇA FUNCIONAL E DO SOLO

TREE RECRUITMENT IN A RESTINGA RAINFOREST: INFLUENCE OF FUNCTIONAL NEIGHBORHOOD AND SOIL 



$$
\begin{gathered}
\text { UNIVERSIDADE DE SÃO PAULO } \\
\text { INSTITUTO DE BIOCIÊNCIAS } \\
\text { PROGRAMA DE PÓS GRADUAÇÃO EM ECOLOGIA }
\end{gathered}
$$

\section{RECRUTAMENTO DE ÁRVORES EM UMA FLORESTA DE RESTINGA: INFLUÊNCIAS DA VIZINHANÇA FUNCIONAL E DO SOLO}

TREE RECRUITMENT IN A RESTINGA RAINFOREST: INFLUENCE OF FUNCTIONAL NEIGHBORHOOD AND SOIL

RENAN LUCAS SIENA DEL BEL

Dissertação apresentada ao Instituto de Blociências da Universidade de São Paulo para obtenção do título de Mestre em Ciências, na área de Ecologia

Orientador: Alexandre Adalardo de Oliveira 


\section{FICHA CATALOGRÁFICA}

Del Bel, Renan Lucas Siena

RECRUTAMENTO DE ÁRVORES EM UMA FLORESTA DE RESTINGA: INFLUÊNCIAS DA VIZINHANÇA FUNCIONAL E DO SOLO / Renan Lucas Siena Del Bel; orientador Alexandre Adalardo de Oliveira -- São Paulo, 2021.

$40 \mathrm{p}$.

Dissertação (Mestrado) -- Instituto de Biociências da Universidade de São Paulo. Programa de Pós-Graduação em Ecologia.

1. Montagem de Comunidades. 2. Atributos Funcionais. 3. Componente de Nicho. 4. Estrutura Fenotípica.

\section{Comissão Julgadora}

$\operatorname{Prof}(\underline{a})$. $\operatorname{Dr}(\underline{a})$.

$\operatorname{Prof}(\underline{a}) . \operatorname{Dr}(\underline{a})$.

$\operatorname{Prof}(\underline{a}) \cdot \operatorname{Dr}(\underline{a})$.

Prof. Dr. Alexandre Adalardo de Oliveira 


\section{AGRADECIMENTOS}

Assinado uma dissertação, há apenas um autor. Mas não se escreve uma única página sozinho.

Em primeiro lugar, gostaria de agradecer à USP, ao IB, ao Departamento de Ecologia e é claro, à CAPES. Sem o suporte dessas instituições o trabalho nem ao menos começaria. Agradeço aos docentes que ajudaram em minha formação, aos discentes, que foram meus companheiros e aos funcionários, que mantém essas instituições funcionando e sem os quais não se faz nada, especialmente Vera Lima, a quem perdi a conta de quantas vezes recorri.

Agradeço aos membros do comitê: Renato, Sara e Kiwi. O trabalho que segue seria bem diferente sem seu input. E junto agradeço Alexandre, que foi meu orientador. Sinto que não seria possível agradecê-los o bastante pela ajuda que deram ao longo do processo e pelo tempo que dedicaram a isso.

Agradeço ao LabTrop e a todos os seus integrantes, passados e presentes, que me vem acolhendo desde antes do início do mestrado. Várias pessoas aqui se destacam: Renan (outro Renan) que sem ele eu estaria perdido num mato sem cachorro, talvez literalmente; Professora Adriana, com alguns toques me deu uma grande ajuda, além de todas as discussões de artigo, aulas, oportunidades de estágio e o bom humor geral que é contagiante; sem Paula e Pamela eu estaria pesando folhas até hoje; Spock, que me mostrou um flamingo magnífico num dia difícil; Jennifer, pelos toques construtivos; e todo o pessoal do recenso da parcela, que tiveram a paciência de me receber no meio do trabalho deles.

Agradeço à minha família e amigos, em especial todos aqueles que dividiram o mesmo teto que eu durante essa jornada. Agradeço à Mônica, que esteve lá do começo ao fim e ao Matheus, Rodolfo e Maria Luísa, que me deram uma mão diretamente com o trabalho, sempre que eu precisei. 
A todos que fizeram parte desse processo, direta ou indiretamente, muito obrigado. 


\section{RESUMO}

Enquanto comunidades biológicas são sistemas complexos, onde inúmeros mecanismos atuam em diferentes escalas e diferentes processos podem gerar 0 mesmo padrão, uma das formas para responder perguntas relativas à montagem de comunidades vegetais é correlacionar padrões ambientais indicativos de processos de nicho subjacentes com os padrões emergentes de uma comunidade. Assim, é essencial que continuemos desenvolvendo nosso entendimento de como representar processos de nicho e como melhor acessar a estrutura da comunidade. Neste trabalho investigamos como diferentes variáveis ambientais contribuem para nosso entendimento dos processos de nicho. Em particular, estamos interessados em como variáveis de natureza biótica ou abiótica interagem e contribuem para o padrão observado na estrutura da comunidade e como a inserção desses componentes é importante para melhor identificar os processos envolvidos na montagem da comunidade. Utilizamos a distribuição espacialmente explícita de atributos foliares dos indivíduos jovens (recrutados nos últimos 10 anos) de uma restinga na llha do Cardoso para representar o padrão resultante dos processos de montagem e utilizamos dados coletados em 2009 para reconstruir o ambiente biótico em que esses indivíduos foram recrutados. Encontramos que diferentes combinações de variáveis ambientais explicam diferentes distribuições de atributos foliares, mas quando incluímos as espécies dos indivíduos jovens como fator aleatório em nossos modelos, nossos resultados não apontam correlação entre variáveis ambientais e atributos foliares. Devido a esses resultados discrepantes inferimos que, embora ambas as variáveis bióticas e abióticas atuem juntas como preditoras da estrutura da comunidade, elas o fazem influenciando a distribuição das espécies, que por sua vez está correlacionada com os valores dos atributos dos indivíduos. Assim, variáveis ambientais são informativas apenas na ausência de informação sobre a identidade das espécies. Baseado nesses resultados, nós propomos que ambos os tipos de variáveis ambientais (bióticas e abióticas) devem ser utilizadas em conjunto para descrever os processos de nicho de uma comunidade, pois ambas podem ser complementares e eventualmente interagir para formar um quadro mais completo. 
Em conjunto, nossos resultados abrem linhas de investigação com potencial para ampliar nosso entendimento dos mecanismos envolvidos na montagem de comunidades de árvores e podem informar futuros trabalhos da área, ajudando-os a obter melhor resolução em análises da estrutura de comunidades.

Palavras chave: Montagem de Comunidades, Atributos Funcionais, Componente de Nicho, Estrutura Fenotípica. 


\section{ABSTRACT}

While biological communities are complex systems in which numerous mechanisms act in different scales and different processes can generate the same pattern, one way to answer questions regarding the assembly of plant communities is to find correlations between environmental patterns indicative of underlying niche processes with the emerging patterns of a community. Because of that, it is essential to keep developing our understanding on how to represent the niche processes and how to better assess the community structure. In this dissertation we investigate how different environmental variables contribute to our understanding of niche processes. In particular, we are interested in how variables of biotic or abiotic nature interact and contribute to the pattern observed in the structure of the community, and whether the integration of these components is important in identifying the processes involved in community assembly. We use the spatially explicit leaf traits distribution of young (recruited in the last 10 years) individuals from a restinga in Ilha do Cardoso to represent the resulting pattern of the assembly processes, as well as data collected in 2009, to rebuild the neighborhood in which these individuals were recruited. We found that different combinations of biotic and abiotic variables explain different leaf traits distributions, but when we include the young individuals species as a random factor in our models, our results point for no correlation between environmental variables and leaf traits. Because of these conflicting results we infer that while both biotic and abiotic variables work together as predictors of the structure of the community, they do so via species distribution, which is in turn, correlated to an individual's leaf traits values. Thus, environmental variables are only informative when we have no information about the identity of the species. Based on our results, we propose that both types of variables (biotic and abiotic) must be used together when trying to assess the niche processes in a community, as both may be complementary and sometimes interact to form a more complete picture. Together our results open some lines of investigation that may further our knowledge over the mechanisms behind the tree community assembly and may inform future work in the field in order to achieve a better resolution in structure analyses. 
Key Words: Community Assembly, Functional Traits, Niche Component, Phenotypic Structure. 


\section{SUMÁRIO}

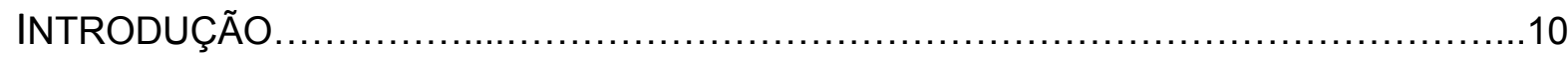

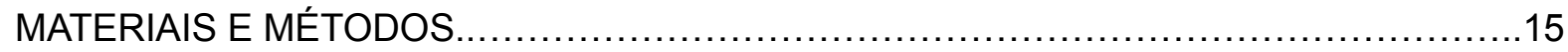

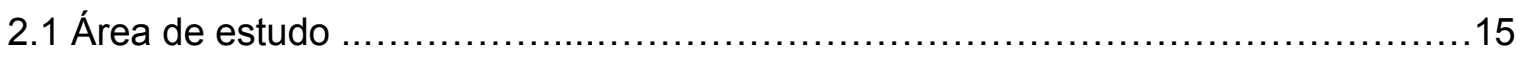

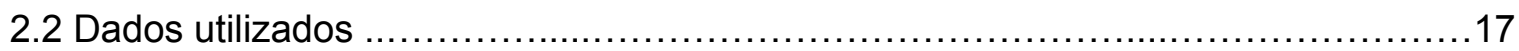

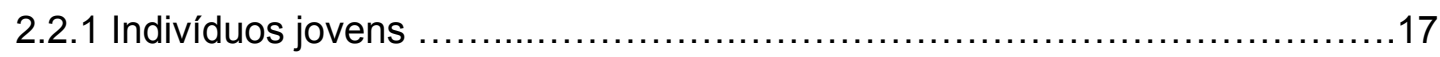

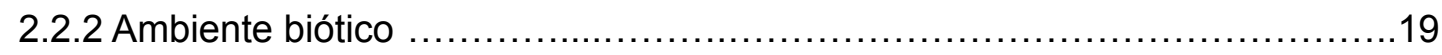

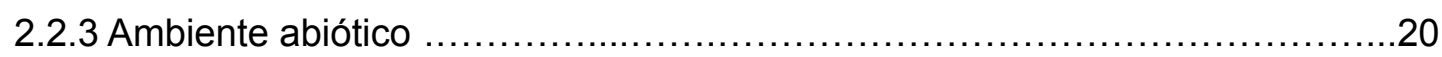

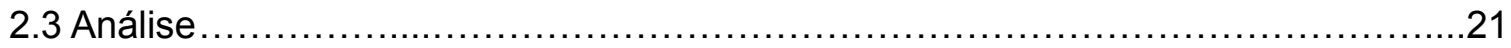

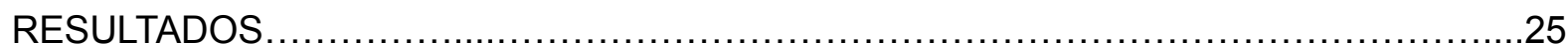

3.1 Com identidade da espécie como variável aleatória.................................

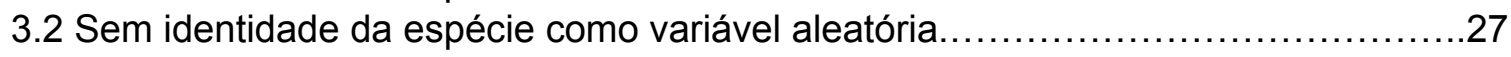

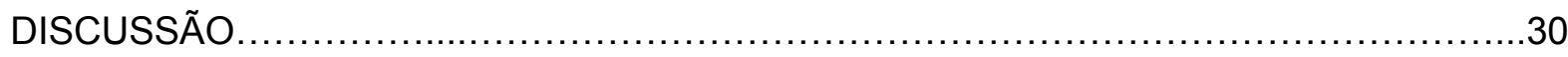

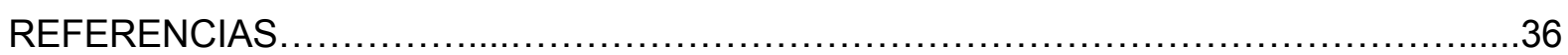




\section{INTRODUÇÃO}

Um dos problemas centrais no estudo de comunidades vegetais, particularmente em florestas tropicais, é determinar quais processos regem a distribuição dos indivíduos. Classicamente se acreditava que, pelo princípio da exclusão competitiva, as populações deveriam dividir o espaço de acordo com pequenas diferenças locais do ambiente (Levin, 1970). Entretanto, foi proposto que o padrão de diversidade e distribuição de espécies que observamos em florestas tropicais poderia ser gerado pelo acaso e por limitações da dispersão de propágulos a partir de sua árvore mãe (Hubbell, 2001). Essa ideia controversa levou a um grande debate no meio científico (Wennekes et al., 2012; Rosindell et al. 2012) que nos trouxe as visões mais atuais do funcionamento das comunidades vegetais, que reconciliam a existência de dinâmicas neutras com dinâmicas determinísticas guiadas pelo ambiente (Hubbell, 2005; Adler et al., 2007; Ovaskainen et al., 2017).

Conforme o debate acerca dos tipos de processos que interferem de forma geral nas comunidade se aproxima de um consenso (Vellend, 2010; Ovaskainen et al., 2017), abrimos caminho para discussões menos amplas, que por sua vez podem levar a um entendimento mais refinado da assembléia de comunidades locais. Por exemplo, dado que processos determinísticos e estocásticos coocorrem, podemos nos perguntar qual a influência de cada um em uma dada escala.

No estudo da montagem de comunidades vegetais, podemos entender "nicho" como o conjunto de variáveis ambientais que influenciam a distribuição dos indivíduos de cada espécie. Nesse caso, a parte da estrutura de uma comunidade que pode ser explicada por variáveis ambientais é o que chamamos de "componente de nicho".

Por muito tempo, trabalhos focados na estrutura da comunidade aproximaram nicho das espécies pelo conjunto das variáveis abióticas (Austin, 2006) e projetos de parcelas permanentes têm investido uma grande quantidade de trabalho em coletar 
essas variáveis. Posteriormente, diversos trabalhos têm focado em interações ao nível de vizinhança, mostrando que caracteres funcionais estão correlacionados com a forma com que plantas interagem com seus vizinhos (eg. Keddy, 1989; Uriarte et al., 2010; Uriarte et al., 2004). Enquanto alguns trabalhos se limitam a usar medidas de proximidade funcional para estimar o efeito de filtro ambiental (e.g. Wang et al., 2016) outros indicam que a forma como as plantas interagem pode ser mais complexa (Kunstler et al., 2016, Kraft et al., 2015) e a importância dessas interações na montagem da comunidade ainda é tema de discussão (Wiegand, 2017). Assim, um dos problemas recorrentes no estudo de comunidades vegetais é como melhor descrever o "componente de nicho", tanto para fins de estudá-lo quanto para fins de entender a relação entre a estrutura da comunidade e o componente de nicho.

Uma pergunta simples que surge é se, ao separarmos as variáveis ambientais em dois grupos: as de natureza biótica e as de natureza abiótica, podemos descrever a importância relativa desses componentes de nicho para chegar a um melhor entendimento da montagem da comunidade.

A questão se baseia na ideia de que os dois tipos de variáveis, biótico e abiótico, não são independentes. Por exemplo, no caso de competição por um certo recurso afetando a distribuição de uma espécie focal, podemos tanto usar a disponibilidade do recurso quanto a presença de competidores como variáveis operacionais, podemos portanto discutir qual captura melhor a realidade e se é preciso ou não considerar a interação entre esses fatores. Em resumo, se a relação entre variáveis ambientais bióticas e abióticas existe, faz sentido perguntar se podemos usar apenas um dos tipos como descritor do componente de nicho, mesmo que saibamos que suas distribuições não sejam perfeitamente sobrepostas.

Embora variáveis bióticas e abióticas possam compartilhar mecanismos pelos quais afetam os seres vivos, elas não são equivalentes. Por exemplo, enquanto inclinação do terreno (abiótico) e cobertura vegetal (biótico) são ambos fatores que podem afetar o crescimento de plantas jovens através de seu efeito na 
disponibilidade de luz (Temps \& Coulson, 1977; Messier, 1998), nenhuma delas prevê sozinha a disponibilidade de luz e cada uma delas também atua como indicadores de outros fatores relevantes, como quantidade de água no solo e intensidade de competição por nutrientes (Moeslund et al., 2013; Canham et al., 2006). Em outro exemplo, a quantidade de matéria orgânica no solo é um reflexo da composição da vizinhança, mas a composição da vizinhança é um reflexo de uma série de fatores e atua sobre diversos mecanismos, enquanto que matéria orgânica no solo é uma medida direta de disponibilidade de recursos e pode ser influenciada por fatores abióticos como a topografia e permeabilidade do solo (Uriarte et al., 2015). Os mecanismos por trás do efeito de cada variável são múltiplos e se sobrepõe a outras variáveis e é por isso que se faz necessária a discussão a respeito de como caracterizar o componente de nicho, para que trabalhemos com as variáveis que melhor resumem a complexa rede de interações de um indivíduo com seu entorno.

Se entendermos que a estrutura de uma comunidade é a distribuição de características dos indivíduos no espaço, podemos nos perguntar se existem padrões gerais nos chamados atributos funcionais foliares. Os atributos funcionais foliares são medidas das folhas de plantas que podemos coletar com relativa facilidade e que são indicadores úteis de suas estratégias ecológicas (Keddy, 1992). Se aceitamos que características do ambiente afetam os indivíduos de uma comunidade, chegando a influenciar as chances de sobrevivência de indivíduos diferentes, é razoável pensar que o ambiente atua selecionando diferentes estratégias ecológicas e, por tanto, que o padrão espacial do ambiente deve ser refletido em um padrão espacial de atributos funcionais (Kraft et al., 2008). Não apenas isso, mas essa abordagem permite visualizar a estrutura de uma comunidade não como a distribuição de uma ampla gama de espécies, mas como a distribuição de poucas variáveis contínuas, o que nos aproxima de encontrar resultados que possam ser interpretados de forma geral (McGill et al., 2006).

Estudos de longo prazo em parcelas permanentes, como a rede ForestGEO (CTFS), permitem não apenas conhecer as distribuição de indivíduos de uma ampla 
área de floresta como também acessar sua trajetória e estado em diferentes momentos. Enquanto um único censo de árvores permite buscar relações entre padrões do presente, o conjunto dos censos ao longo de anos permite observar fenômenos e relacionar padrões do presente a eventos anteriores. Por exemplo, ao incluirmos informação do momento em que indivíduos se estabeleceram na comunidade, podemos considerar que indivíduos mais recentes não devem ter influenciado no crescimento e nascimento dos mais antigos e, ao mesmo tempo, podemos relacionar os padrões de distribuição dos indivíduos mais recentes ao padrão dos indivíduos que são mais antigos.

Uma das dificuldades em avaliar como a estrutura da comunidade responde a variáveis bióticas é que a estrutura da comunidade pode ser considerada em si mesma uma variável biótica, por exemplo, os atributos funcionais de uma vizinhança podem estar respondendo aos atributos funcionais dessa mesma vizinhança. Entretanto, ao separarmos os indivíduos temporalmente, podemos considerar que as árvores mais antigas, independente dos processos que geraram sua distribuição atual, fizeram parte do ambiente dos indivíduos mais novos enquanto estes ainda se estabeleciam na comunidade. Dessa forma, para cada indivíduo incluído apenas no censo mais recente, ou seja, apenas os indivíduos recém estabelecidos, estabelecemos como vizinhança os indivíduos que estiveram presentes no censo imediatamente anterior na área ao redor da localização do indivíduo focal. Além de evitamos a autocorrelação entre variáveis bióticas, essa abordagem torna mais simples investigar algumas hipóteses a respeito dos processos que afetam a estrutura da comunidade, pois enquanto a comunidade como um todo é resultado de complexos processos históricos, atuando em largas escalas espaciais e temporais, a distribuição de recrutas é resultado de eventos recentes e localmente relevantes, aos quais nós, em teoria, podemos ter acesso.

Dessa forma, neste estudo iremos avaliar como os atributos funcionais foliares de indivíduos recrutados em uma floresta de restinga respondem ao seu ambiente. Para isso, vamos primeiro estabelecer se as variáveis bióticas e abióticas podem atuar 
como preditores da distribuição espacial desses atributos e, concomitantemente investigar se o uso desses dois tipos de variáveis é redundante ou complementar no contexto de compreender padrões na estrutura da comunidade de árvores. 


\section{MATERIAIS E MÉTODOS}

\section{1 Área de estudo}

A parcela permanente está localizada no Parque Estadual da Ilha do Cardoso (PEIC), no município de Cananéia - SP, latitude 2504'41"S, longitude 47055'53"O (Figura 1). A parcela compreende uma floresta alta de restinga, o terreno é plano, variando entre 3 e 7 metros acima do nível do mar. O solo abaixo da vegetação é arenoso e ácido, podendo ser dividido em dois tipos principais, espodossolo e neossolo, sendo o neossolo presente em área mais baixa e mais próxima ao mar (Figura 2). A área de neossolo também coincide com uma área que enfrenta alagamentos periódicos. Informações detalhadas a respeito da caracterização do solo podem ser encontradas em Gomes et al. (2007).

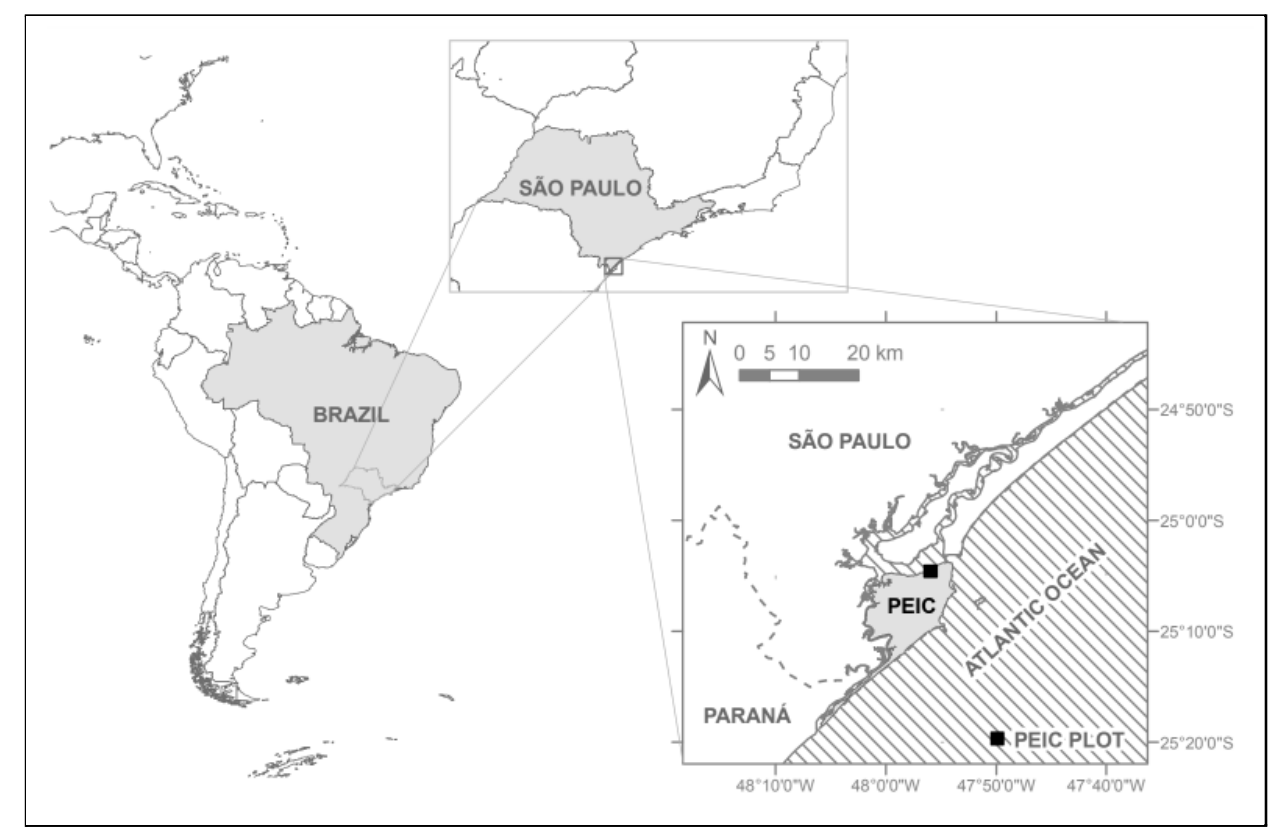

Fig. 1 Localização da parcela permanente (PEIC PLOT) em relação à América Latina. Modificado de Lima et al.,2011. 


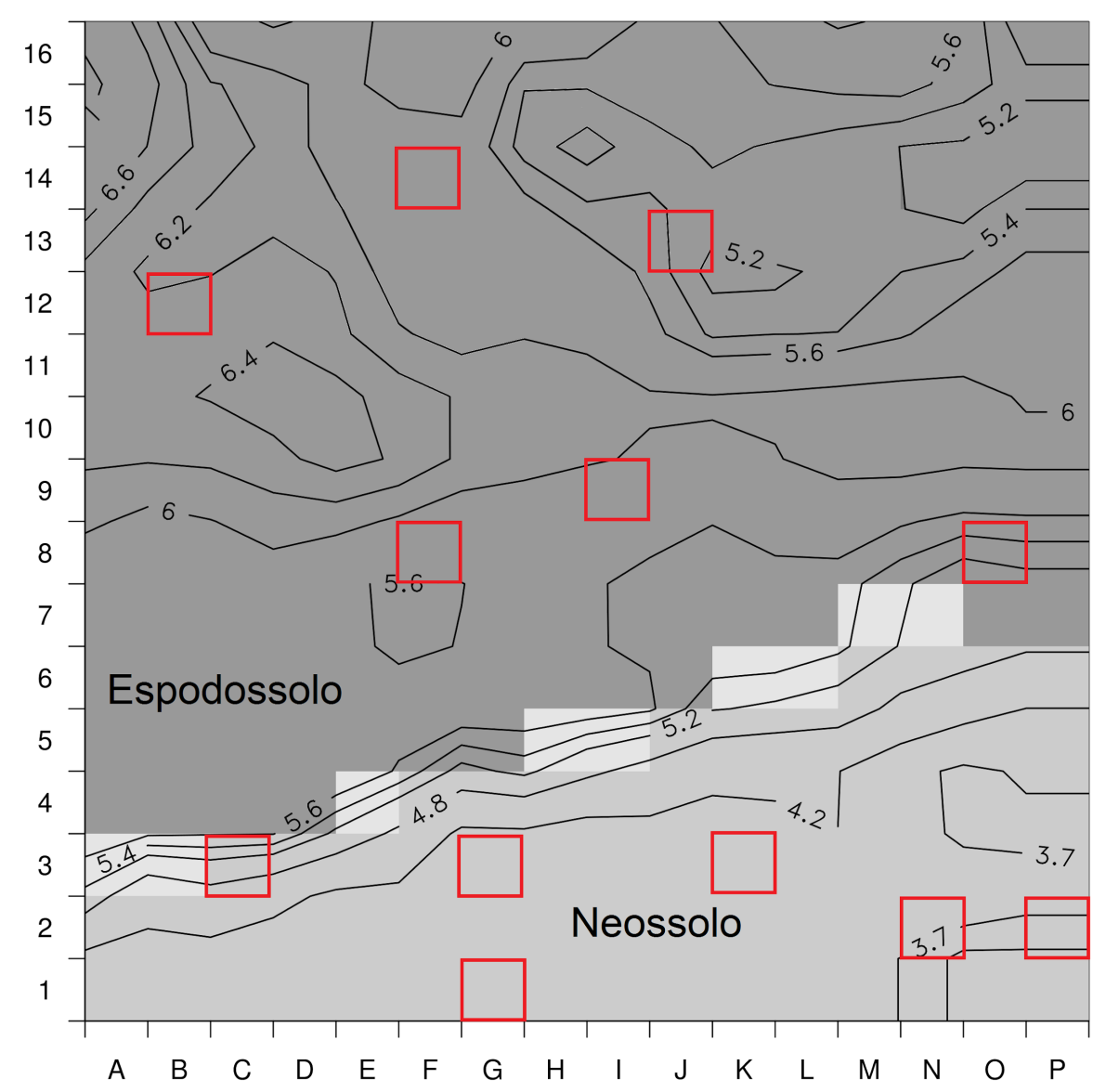

Fig. 2 Mapa da parcela permanente apresentando os dois tipos de solo (espodossolo e neossolo) por tons diferentes de cinza, para cada sub-parcela. $\mathrm{O}$ tom mais claro de cinza que ocorre na intersecção dos dois principais foi considerado como tipo de solo transicional. As curvas de nível representam a altura em relação ao nível do mar em metros. As sub-parcelas destacadas em vermelho foram as utilizadas no presente estudo. Cada sub-parcela tem 20 metros de lado. Modificado de Oliveira et al., 2014.

A parcela, demarcada em 2002, é um quadrado de 320 m de lado subdividido em 256 sub-parcelas de 20 × 20 m. A partir de 2009 a parcela foi cadastrada junto ao ForestGeo (antigo CTFS) ${ }^{1}$, após mudar os protocolos de censo para seguir os padrões estabelecidos por Condit (1998). O censo mais recente foi finalizado em 2019 e o anterior em 2009.

\footnotetext{
${ }^{1}$ Mais informações disponíveis no site https://forestgeo.si.edu/
} 


\subsection{Dados utilizados}

\subsubsection{Indivíduos jovens}

O censo de 2019 nos permitiu conhecer a localização e identificação de todos os indivíduos que atingiram o tamanho mínimo de $1 \mathrm{~cm}$ de diâmetro à altura do peito (altura padronizada como 1,30 m a partir do solo) entre 2009 e 2018. Parte desses novos indivíduos tiveram seus atributos funcionais foliares coletados. Para isso, foram sorteadas 12 sub-parcelas, sendo 6 em neossolo e 6 em espodossolo, com o cuidado de evitar sub-parcelas adjacentes para que não haja sobreposição entre as vizinhanças dos indivíduos novos de parcelas diferentes (Figura 2). Todos os indivíduos novos dentro das sub-parcelas sorteadas foram incluídos para a coleta de atributos funcionais, com exceção de indivíduos com poucas folhas, devido ao impacto da coleta, e indivíduos das ordens Arecales e Cyatheales, pois tem hábito de vida palmoide e suas folhas podem apresentar valores de atributos funcionais muito diferentes dos encontrados em outros grupos, sendo difícil sua comparação direta. Ao todo foram amostrados 299 indivíduos pertencentes a 67 espécies.

Para cada indivíduo amostrado, foram coletadas 3 folhas de ramos diferentes. Cada folha foi hidratada e pesada (peso úmido), escaneada, desidratada e pesada novamente (peso seco). A área foliar foi obtida através do software de processamento de imagens ImageJ (Schneider et al., 2012), usando as imagens escaneadas. Com essas informações estimamos valores médios de área específica da folha (SLA), área foliar (LA) e conteúdo de massa seca da folha (LDMC) para cada indivíduo jovem. O protocolo de coleta seguiu as orientações de Cornelissen et al. (2003), onde pode-se encontrar mais detalhes do procedimento.

Os valores de atributos funcionais dos indivíduos apresentam diferentes distribuições em cada espécie. Em comparações par-a-par espécies parecem ter tendências bastante distintas no geral, mas devido a intersecções nos valores de 
espécies com tendências mais próximas, no conjunto os valores de atributos formam um contínuo (Figura 3).

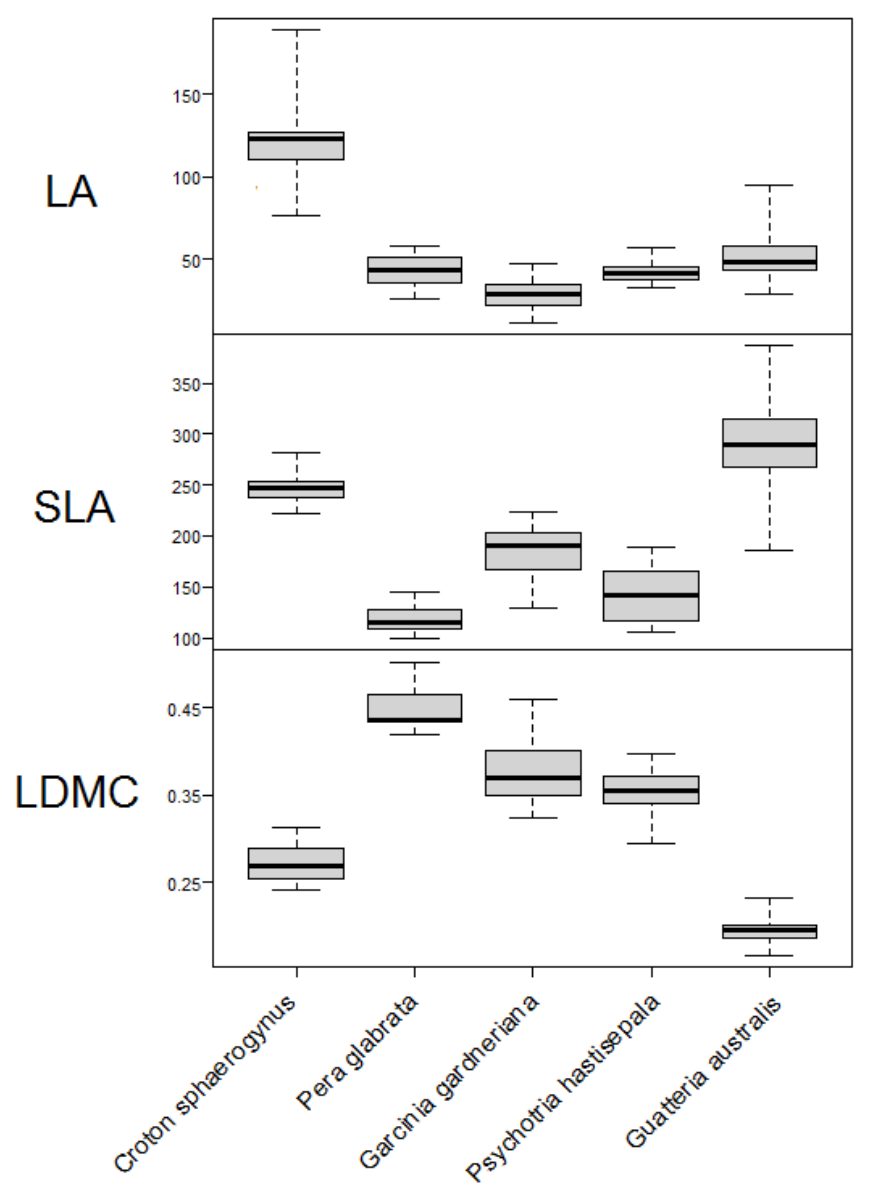

Fig. 3 Distribuições dos valores de atributo funcional dos indivíduos jovens, exemplificado pelas 5 espécies com mais indivíduos coletados (mais do que 10 indivíduos cada). LA em cm², $\mathrm{SLA}$ em $\mathrm{cm}^{2} / \mathrm{g}$ e LDMC em valor de 0 a 1.

Quando combinamos os dados de todas as espécies, as distribuições dos valores de SLA e LA apresentam uma forma relativamente simétrica ao redor da média, mas com uma longa cauda de valores altos. Por isso, transformamos esses valores pela operação logarítmica (chamados LSLA e LLA, respectivamente), para que essa grande disparidade seja interpretada em termos de ordens de grandeza e para que as análises estatísticas possam usar ferramentas mais gerais. 


\subsubsection{Ambiente biótico}

A localização relativa (com precisão em decímetros) e identidade da espécie dos indivíduos adultos foram obtidas dos dados do censo de 2009 da parcela permanente. Todos os indivíduos arbóreos com diâmetro à altura do peito maior que $1 \mathrm{~cm}$ foram identificados taxonomicamente, tiveram a localização registrada em coordenadas relativas aos limites da parcela, medidos quanto ao diâmetro à altura do peito e quanto à altura da copa e tiveram o número de fustes contados. Além disso, todas as espécies na parcela foram amostradas para medição de atributos funcionais.

Determinamos que o ambiente biótico de um indivíduo seria considerado como as características da vizinhança dentro de um raio de $10 \mathrm{~m}$ em 2009. Estamos cientes que a vizinhança efetiva de um indivíduo pode se estender além de $10 \mathrm{~m}$ de raio, a depender das espécies envolvidas (Uriarte, 2004). Entretanto, aumentar o raio da vizinhança nos impediria de capturar as diferenças entre regiões da parcela e criaria uma autocorrelação espacial entre indivíduos em sub-parcelas diferentes, já que isto criaria intersecções entre as vizinhanças. Dado que a influência de um indivíduo em outro é inversamente proporcional à sua distância (Uriarte, 2004), escolhemos trabalhar com um raio que deve incluir os indivíduos mais relevantes da vizinhança sem que se torne excessivamente extenso.

As características da vizinhança são dadas pela soma da área basal de coespecíficos e pelos valores médios de SLA, LA, LDMC e densidade da madeira (WD) na vizinhança. O valor médio de cada atributo funcional de uma vizinhança foi calculado ao ponderar as médias de cada espécie da parcela pelo número exato de representantes dessa espécie dentro do raio da vizinhança, excluídos coespecíficos do indivíduo focal.

Dado que efeitos que favoreçam maior agregamento de determinada espécie não necessariamente estarão relacionados a seus atributos funcionais foliares (por exemplo a limitação de dispersão) e assumindo que os atributos funcionais de cada 
indivíduos assumem valores em torno da média de sua espécie, condições que favorecem certas espécies podem criar uma tendência nos atributos da vizinhança. Dessa forma, quando separamos coespecíficos de não coespecíficos no cálculo de valor médio dos atributos da vizinhança evitamos que o valor médio de um atributo na vizinhança seja enviesado para ser mais semelhante ao valor do indivíduo focal. Além disso, entendemos que a densidade de coespecíficos representa parte dos processos que não são capturados diretamente por outras informações da vizinhança, como a competição intraespecífica (Adler et. al, 2018).

\subsubsection{Ambiente abiótico}

O ambiente abiótico foi caracterizado por medições químicas do solo em cada sub-quadrado da parcela. Incluímos medições da concentração dos elementos P, K, $\mathrm{Mg}, \mathrm{Ca}, \mathrm{Na}$, Al e H, da soma de bases (SB), matéria orgânica (MO), porcentagem de saturação de bases (V\%), pH em solução de $\mathrm{CaCl}_{2}$ e $\Delta \mathrm{pH}$. Essas medidas representam a presença e disponibilidade de macro e micronutrientes e possíveis fatores de estresse (como presença de alumínio trocável) que podem influenciar no desenvolvimento de plantas (Chapman, 1966). Utilizamos medições em duas faixas de profundidades: De 0 a $5 \mathrm{~cm}$ e de 5 a $25 \mathrm{~cm}$, profundidade compatível com a profundidade de raízes na restinga (Bonilha et al., 2004). Para cada sub-quadrado da parcela, o valor de uma variável é dado pela média de três pontos de amostragem. Ao invés de utilizar todas as variáveis, fizemos uma análise de componentes principais e utilizamos os cinco primeiros componentes como resumo para caracterizar o solo. Esses cinco componentes resumem $68.8 \%$ de toda a variação e a parte da variância explicada por cada componente pode ser encontrada na Figura 3. Além disso, utilizamos também a divisão do solo em neossolo e espodossolo, por sub-parcela. 


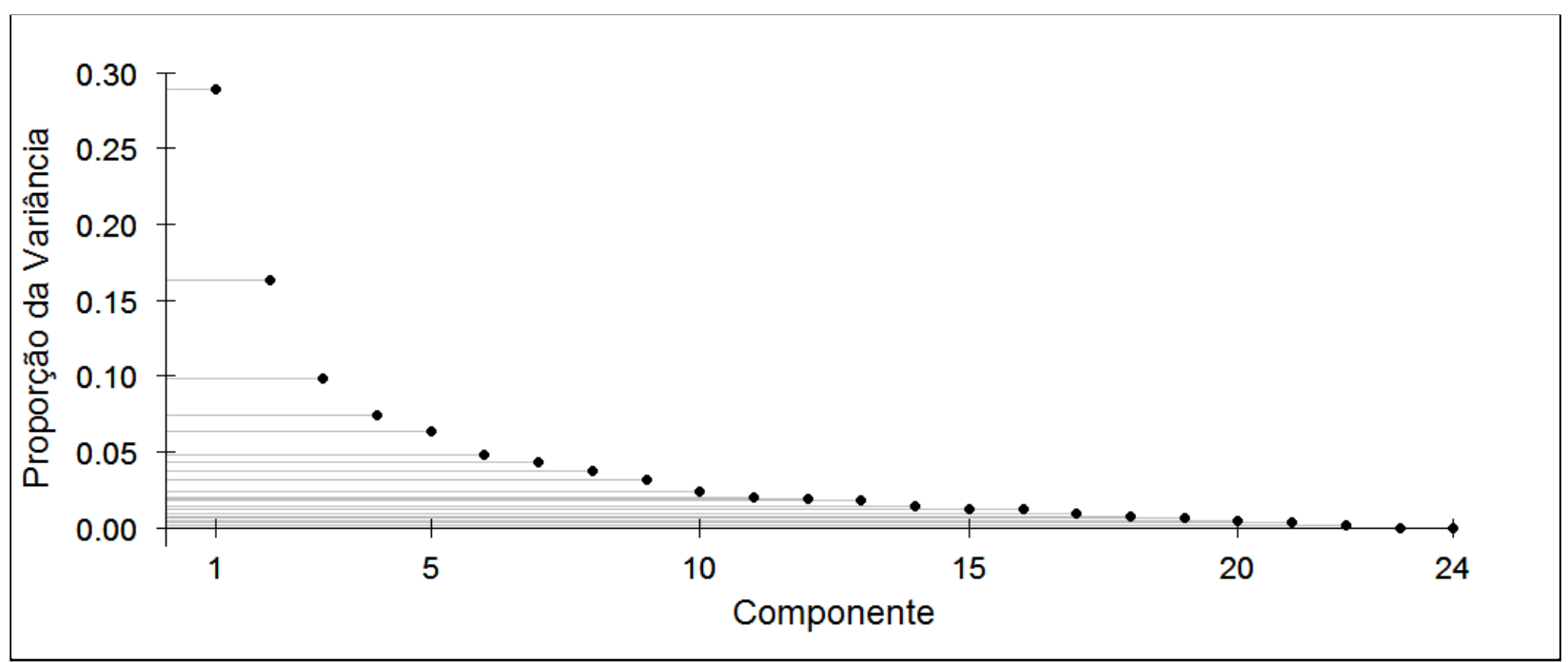

Fig. 3 Proporção da variância (em escala de 0 a 1) explicada por cada componente principal das variáveis de solo. Note que o quinto componente é o último que adiciona mais do que $5 \%$ de explicação e que os intervalos entre um componente tendem a diminuir, mas que o intervalo entre os componentes 5 e 6 é maior do que entre 5 e 4.

\subsection{Análise}

Criamos uma série de modelos lineares mistos (tabela 1) usando como variáveis preditoras as variáveis abióticas ( 5 componentes principais das medições de solo), variáveis bióticas (média dos atributos funcionais da vizinhança e área basal dos coespecíficos) e o tipo de solo (neossolo ou espodossolo). Como variáveis resposta utilizamos os valores dos atributos funcionais dos recrutas.

O tipo de solo foi tratado separado de variáveis abióticas pois atua como um resumo de diversas variáveis bióticas e abióticas que não foram diretamente coletadas para este trabalho. Notavelmente os tipos de solo diferem quanto à composição física do solo, propensão a alagamento e prevalência de bromélias terrestres. Da mesma forma, a presença de indivíduos mais velhos, da mesma espécie que o indivíduo focal, foi desacoplada das outras variáveis bióticas nos modelos, pois pode representar uma força de competição mais intensa do que com outras espécies e ao mesmo tempo é um indicador de uma área adequada para o crescimento desta espécie (através de fatores que não necessariamente 
mensuramos). Além de modelos que incluem as diferentes combinações lineares dessas variáveis, incluímos também um modelo onde o primeiro componente principal do solo interage com as variáveis bióticas e um modelo nulo, onde desvios da média são atribuídos ao acaso. Cada um dos modelos foi ajustado três vezes, cada vez com uma variável resposta diferente (LLA, LSLA e LDMC), totalizando em 3 conjuntos de 14 modelos. Um resumo de todos os modelos criados e suas variáveis pode ser encontrado na Tabela 1.

Pretendemos capturar as tendências gerais nos valores de atributos funcionais, mas esta ideia pode ser interpretada de duas maneiras: No primeiro caso entendemos que o efeito do ambiente sobre os atributos atua em cada espécie, mas que a distribuição das espécies pode ser reflexo de outras dinâmicas. Nesse caso, por exemplo, em uma área que favoreça maior SLA, observamos que os indivíduos encontrados têm SLA acima da média para sua espécie. Tratamos esse primeiro caso em nossa análise ao incluir a identidade da espécie do indivíduo focal como variável aleatória em cada um dos modelos, de modo que valores de atributos são tratados no relativo à sua espécie.

O segundo caso seria onde procuramos tendências absolutas nos valores de atributos. Nesse caso, em uma região que favoreça maior SLA, encontraremos indivíduos com SLA acima da média da parcela. Para explorar esse caso, fizemos uma segunda bateria de testes onde não incluímos a identidade da espécie do indivíduo focal nos modelos. Esperamos que as duas baterias de modelos, em conjunto, apresentem um quadro mais completo dos processos ocorrendo na comunidade.

Os diferentes modelos foram comparados via Critério de Informação de Akaike (AIC), para entender como cada tipo de variável melhora nossa capacidade de explicar os dados e para selecionar o modelo mais plausível perante o padrão dos dados. É esperado que o ambiente biótico e abiótico imponham vetores de seleção sobre os indivíduos, mas não sabemos dizer se as variáveis bióticas e abióticas utilizadas contribuem juntas para um modelo mais plausível das tendências nos 
valores de atributos funcionais dos indivíduos ou se modelos mais simples oferecem explicações mais parcimoniosas.

Tabela 1 - Tipos de modelos construídos para testar a contribuição de diferentes variáveis ambientais para a predição dos valores de atributos funcionais de indivíduos jovens. $A=$ conjunto das variáveis abióticas ${ }^{2} ; B=$ componente heteroespecífico das variáveis bióticas ${ }^{3}$; PC1 = Primeiro componente principal das medidas de composição do solo, incluso em A; C = Soma da área basal de coespecíficos na vizinhança; $\mathrm{T}=$ tipo de solo.

\begin{tabular}{|c|c|}
\hline Modelo & Variáveis Preditoras \\
\hline Modelo nulo & - \\
\hline Influência abiótica & $A$ \\
\hline Influência biótica & $(B+C)$ \\
\hline $\begin{array}{r}\text { Influência biótica apenas por } \\
\text { coespecíficos }\end{array}$ & C \\
\hline Influência biótica e abiótica & $A+(B+C)$ \\
\hline $\begin{array}{l}\text { Influência biótica apenas por } \\
\text { coespecíficos e abiótica }\end{array}$ & $A+C$ \\
\hline Interação biótico-abiótico & $A+(B+C)+P C 1:(B+C)$ \\
\hline Influência do tipo de solo & $\mathrm{T}$ \\
\hline $\begin{array}{r}\text { Influência abiótica com tipo de } \\
\text { solo }\end{array}$ & $A+T$ \\
\hline $\begin{array}{r}\text { Influência biótica com tipo de } \\
\text { solo }\end{array}$ & $(B+C)+T$ \\
\hline $\begin{array}{l}\text { Influência biótica apenas por } \\
\text { coespecíficos com tipo de solo }\end{array}$ & $\mathrm{C}+\mathrm{T}$ \\
\hline $\begin{array}{r}\text { Influência biótica e abiótica } \\
\text { com tipo de solo }\end{array}$ & $A+(B+C)+T$ \\
\hline $\begin{array}{r}\text { Influência biótica apenas por } \\
\text { coespecíficos e abiótica com } \\
\text { tipo de solo }\end{array}$ & $A+C+T$ \\
\hline $\begin{array}{r}\text { Interação biótico-abiótico } \\
\text { com tipo de solo }\end{array}$ & $A+(B+C)+P C 1:(B+C)+T$ \\
\hline
\end{tabular}

\footnotetext{
${ }^{2}$ Cinco primeiros componentes principais das variáveis químicas do solo

${ }^{3}$ Valores médios de SLA, LA, LDMC e WD da vizinhança
} 
Para comparar o poder de explicação dos modelos utilizamos pseudo- $R^{2}$ (Nakagawa, 2017), que separa a parcela da variância dos dados que é explicada pelo conjunto das variáveis fixas e aleatórias ( $R^{2}$ condicional), da variância que é explicada apenas pelas variáveis fixas ( $R^{2}$ marginal), esse método tem a vantagem de ser interpretado de forma análoga ao $\mathrm{R}^{2}$ convencional e de permitir acessar a explicação dada pelas variáveis fixas. 


\section{RESULTADOS}

\subsection{Com identidade da espécie como variável aleatória}

Para os três atributos utilizados, encontramos o mesmo padrão geral: Modelos com menos parâmetros são mais prováveis que modelos mais cheios e, nos três casos, o suporte para o modelo nulo é consideravelmente maior do que para o segundo modelo mais provável, o modelo de influência do tipo de solo.

Através da análise pseudo- $\mathrm{R}^{2}$, encontramos que em LLA e LDMC a variância é explicada, quase que em sua totalidade (entre 0.82 e 0.94), pelas variáveis aleatórias. Em LSLA o $\mathrm{R}^{2}$ marginal chegou a 0.037 no modelo mais cheio, o mesmo modelo apresenta $\mathrm{R}^{2}$ condicional de 0.776 . Os resultados detalhados, incluindo valor de AIC e pseudo- $R^{2}$ para todos os modelos, podem ser encontrados na Tabela 2.

Tabela 2 - Resultados dos modelos lineares mistos que incluem a identidade das espécies como variável aleatória. Em negrito o modelo mais plausível para cada variável resposta ( $\triangle A I C>2$ ). Os modelos foram agrupados por variável resposta, com o cabeçalho reproduzido antes de cada grupo para facilitar a compreensão. Note que a tabela está dividida entre esta página e a próxima.

\begin{tabular}{|c|c|c|c|c|}
\hline Modelo & $\begin{array}{l}\text { Número de } \\
\text { parâmetros }\end{array}$ & AIC & $\mathbf{R}^{\mathbf{2}}$ marginal & $\mathbf{R}^{2}$ condicional \\
\hline LLA 1 & 3 & 370.41 & 0.000 & 0.935 \\
\hline $\mathrm{LLA} \sim \mathrm{T}$ & 4 & 374.87 & 0.001 & 0.934 \\
\hline LLA $\sim \mathrm{C}$ & 4 & 389.16 & 0.000 & 0.935 \\
\hline LLA $\sim \mathrm{C}+\mathrm{T}$ & 5 & 393.60 & 0.001 & 0.934 \\
\hline LLA $\sim A$ & 8 & 405.85 & 0.004 & 0.935 \\
\hline $\mathrm{LLA} \sim \mathrm{A}+\mathrm{T}$ & 9 & 411.29 & 0.004 & 0.934 \\
\hline LLA $(B+C)$ & 8 & 412.24 & 0.001 & 0.935 \\
\hline $\operatorname{LLA} \sim(B+C)+T$ & 9 & 417.43 & 0.002 & 0.934 \\
\hline $\mathrm{LLA} \sim \mathrm{A}+\mathrm{C}$ & 9 & 424.52 & 0.004 & 0.934 \\
\hline LLA $\sim A+C+T$ & 10 & 429.95 & 0.004 & 0.934 \\
\hline$L L A \sim A+(B+C)$ & 13 & 448.22 & 0.005 & 0.935 \\
\hline$L L A \sim A+(B+C)+T$ & 14 & 453.47 & 0.005 & 0.934 \\
\hline$L L A \sim A+(B+C)+(B+C): P C 1$ & 18 & 496.99 & 0.007 & 0.936 \\
\hline$L L A \sim A+(B+C)+(B+C): P C 1+T$ & 19 & 501.92 & 0.007 & 0.937 \\
\hline
\end{tabular}




\begin{tabular}{|c|c|c|c|c|}
\hline Modelo & $\begin{array}{l}\text { Número de } \\
\text { parâmetros }\end{array}$ & AIC & $\mathbf{R}^{\mathbf{2}}$ marginal & $\mathbf{R}^{2}$ condicional \\
\hline LSLA 1 & 3 & -76.16 & 0.000 & 0.791 \\
\hline LSLA T & 4 & -70.89 & 0.003 & 0.787 \\
\hline LSLA $\sim \mathrm{C}$ & 4 & -56.13 & 0.000 & 0.790 \\
\hline LSLA $\sim \mathrm{C}+\mathrm{T}$ & 5 & -50.87 & 0.003 & 0.786 \\
\hline $\operatorname{LSLA} \sim(B+C)$ & 8 & -47.10 & 0.024 & 0.787 \\
\hline LSLA A & 8 & -41.78 & 0.022 & 0.780 \\
\hline $\operatorname{LSLA} \sim(B+C)+T$ & 9 & -40.55 & 0.025 & 0.784 \\
\hline LSLA $\sim A+T$ & 9 & -34.84 & 0.022 & 0.779 \\
\hline LSLA $\sim A+C$ & 9 & -21.77 & 0.022 & 0.778 \\
\hline $\mathrm{LSLA} \sim \mathrm{A}+\mathrm{C}+\mathrm{T}$ & 10 & -14.84 & 0.022 & 0.777 \\
\hline LSLA A + $(B+C)$ & 13 & -3.00 & 0.033 & 0.779 \\
\hline LSLA $\sim A+(B+C)+T$ & 14 & 3.55 & 0.033 & 0.778 \\
\hline LSLA A + (B+C) + (B+C):PC1 & 18 & 56.73 & 0.037 & 0.777 \\
\hline \multirow[t]{2}{*}{$L S L A \sim A+(B+C)+(B+C): P C 1+T$} & 19 & 63.13 & 0.037 & 0.776 \\
\hline & $\begin{array}{l}\text { Número de } \\
\text { parâmetros }\end{array}$ & AIC & $\mathbf{R}^{2}$ marginal & $\mathbf{R}^{2}$ condicional \\
\hline LDMC 1 & 3 & -1042.44 & 0.000 & 0.824 \\
\hline LDMC $\sim \mathrm{T}$ & 4 & -1032.50 & 0.001 & 0.823 \\
\hline $\mathrm{LDMC} \sim \mathrm{C}$ & 4 & -1019.15 & 0.000 & 0.824 \\
\hline $\mathrm{LDMC} \sim \mathrm{C}+\mathrm{T}$ & 5 & -1009.24 & 0.001 & 0.822 \\
\hline $\mathrm{LDMC} \sim \mathrm{A}$ & 8 & -981.78 & 0.005 & 0.825 \\
\hline LDMC $(B+C)$ & 8 & -980.81 & 0.007 & 0.831 \\
\hline $\mathrm{LDMC} \sim \mathrm{A}+\mathrm{T}$ & 9 & -971.60 & 0.005 & 0.825 \\
\hline LDMC $\sim(B+C)+T$ & 9 & -970.57 & 0.007 & 0.829 \\
\hline LDMC $\sim A+C$ & 9 & -958.59 & 0.006 & 0.824 \\
\hline LDMC $\sim \mathrm{A}+\mathrm{C}+\mathrm{T}$ & 10 & -948.41 & 0.006 & 0.824 \\
\hline LDMC $\sim A+(B+C)$ & 13 & -919.44 & 0.009 & 0.831 \\
\hline $\mathrm{LDMC} \sim \mathrm{A}+(\mathrm{B}+\mathrm{C})+\mathrm{T}$ & 14 & -909.67 & 0.009 & 0.831 \\
\hline$L D M C \sim A+(B+C)+(B+C): P C 1$ & 18 & -843.54 & 0.012 & 0.835 \\
\hline LDMC $\sim A+(B+C)+(B+C): P C 1+T$ & 19 & -833.76 & 0.012 & 0.835 \\
\hline
\end{tabular}




\subsection{Sem identidade da espécie como variável aleatória}

Em nossa segunda bateria de testes, diferentes modelos foram selecionados como mais plausíveis para cada variável resposta. Em todos os casos foram selecionados modelos com variáveis bióticas em conjunto com tipo de solo e/ou variáveis abióticas. Os resultados detalhados, incluindo o valor de AIC e $\mathrm{R}^{2}$ para todos os modelos, podem ser encontrados na Tabela 3.

Para LLA, o modelo mais plausível foi o modelo de influência biótica apenas por coespecíficos e abiótica com tipo de solo, com $\mathrm{R}^{2}$ de 0.207 . O segundo mais plausível foi a interação biótico-abiótico com tipo de solo ( $\triangle \mathrm{AIC}$ de 3.38 ), e todos os modelos que incluíram tipo de solo foram considerados mais plausíveis que os modelos sem tipo de solo.

Tabela 3 - Resultados dos modelos lineares mistos que não incluem a identidade das espécies como variável aleatória. Em negrito o modelo mais plausível para cada variável resposta ( $\triangle A I C>2$ ). Os modelos foram agrupados por variável resposta, com o cabeçalho reproduzido antes de cada grupo para facilitar a compreensão. Note que a tabela está dividida entre esta página e a próxima.

\begin{tabular}{|c|c|c|c|}
\hline Modelo & $\begin{array}{l}\text { Número de } \\
\text { parâmetros }\end{array}$ & AIC & $\mathbf{R}^{2}$ \\
\hline$L L A \sim A+(B+C)+T$ & 13 & 818.40 & 0.207 \\
\hline$L L A \sim A+(B+C)+(B+C): P C 1+T$ & 18 & 821.78 & 0.221 \\
\hline $\mathrm{LLA} \sim \mathrm{A}+\mathrm{C}+\mathrm{T}$ & 9 & 836.56 & 0.138 \\
\hline LLA $\sim \mathrm{C}+\mathrm{T}$ & 4 & 838.53 & 0.105 \\
\hline $\operatorname{LLA} \sim(B+C)+T$ & 8 & 839.41 & 0.125 \\
\hline LLA $\sim A+T$ & 8 & 840.92 & 0.120 \\
\hline LLA $\sim \mathrm{T}$ & 3 & 843.99 & 0.083 \\
\hline$L L A \sim A+(B+C)$ & 12 & 848.00 & 0.121 \\
\hline LLA $\sim A+C$ & 8 & 848.57 & 0.098 \\
\hline$L L A \sim A+(B+C)+(B+C): P C 1$ & 17 & 849.35 & 0.144 \\
\hline LLA $\sim A$ & 7 & 852.68 & 0.079 \\
\hline LLA $\sim \mathrm{C}$ & 3 & 862.56 & 0.024 \\
\hline $\mathrm{LLA} \sim(\mathrm{B}+\mathrm{C})$ & 7 & 862.61 & 0.049 \\
\hline LLA $\sim 1$ & 2 & 867.68 & 0.000 \\
\hline
\end{tabular}




\begin{tabular}{|c|c|c|c|}
\hline Modelo & $\begin{array}{l}\text { Número de } \\
\text { parâmetros }\end{array}$ & AIC & $\mathbf{R}^{2}$ \\
\hline$L S L A \sim A+(B+C)$ & 12 & 138.44 & 0.196 \\
\hline$L S L A \sim A+(B+C)+T$ & 13 & 138.47 & 0.200 \\
\hline LSLA $\sim A+(B+C)+(B+C): P C 1+T$ & 18 & 143.04 & 0.211 \\
\hline LSLA $\sim A+(B+C)+(B+C): P C 1$ & 17 & 143.29 & 0.206 \\
\hline LSLA $\sim A+C$ & 8 & 147.22 & 0.152 \\
\hline LSLA $(B+C)+T$ & 8 & 148.58 & 0.148 \\
\hline LSLA $\sim A+C+T$ & 9 & 149.20 & 0.152 \\
\hline $\operatorname{LSLA} \sim(B+C)$ & 7 & 156.28 & 0.121 \\
\hline LSLA $\sim \mathrm{C}+\mathrm{T}$ & 4 & 160.63 & 0.091 \\
\hline LSLA A & 7 & 165.22 & 0.094 \\
\hline LSLA $\sim A+T$ & 8 & 167.21 & 0.094 \\
\hline LSLA C & 3 & 167.37 & 0.064 \\
\hline LSLA $\sim \mathrm{T}$ & 3 & 178.68 & 0.028 \\
\hline LSLA 1 & 2 & 185.13 & 0.000 \\
\hline Modelo & $\begin{array}{l}\text { Número de } \\
\text { parâmetros }\end{array}$ & AIC & $\mathbf{R}^{2}$ \\
\hline LDMC $(B+C)+T$ & 8 & -668.30 & 0.103 \\
\hline $\mathrm{LDMC} \sim \mathrm{A}+(\mathrm{B}+\mathrm{C})+\mathrm{T}$ & 13 & -665.97 & 0.124 \\
\hline LDMC $\sim \mathrm{C}+\mathrm{T}$ & 4 & -664.62 & 0.069 \\
\hline $\mathrm{LDMC} \sim \mathrm{A}+(\mathrm{B}+\mathrm{C})+(\mathrm{B}+\mathrm{C}): \mathrm{PC} 1+\mathrm{T}$ & 18 & -662.55 & 0.140 \\
\hline LDMC $\sim \mathrm{C}$ & 3 & -662.04 & 0.055 \\
\hline $\mathrm{LDMC} \sim \mathrm{A}+(\mathrm{B}+\mathrm{C})$ & 12 & -660.53 & 0.103 \\
\hline $\mathrm{LDMC} \sim \mathrm{A}+\mathrm{C}$ & 8 & -659.15 & 0.076 \\
\hline LDMC (B+C) & 7 & -658.70 & 0.069 \\
\hline LDMC $\sim A+C+T$ & 9 & -657.97 & 0.078 \\
\hline $\mathrm{LDMC} \sim \mathrm{A}+(\mathrm{B}+\mathrm{C})+(\mathrm{B}+\mathrm{C}): \mathrm{PC} 1$ & 17 & -656.16 & 0.117 \\
\hline LDMC T & 3 & -649.49 & 0.015 \\
\hline LDMC 1 & 2 & -646.93 & 0.000 \\
\hline LDMC $\sim A$ & 7 & -643.59 & 0.022 \\
\hline LDMC $\sim \mathrm{A}+\mathrm{T}$ & 8 & -642.39 & 0.024 \\
\hline
\end{tabular}

Para LSLA, os modelos de influência biótica e abiótica - com e sem tipo de solo - ficaram empatados como modelo mais plausível e apresentam $R^{2}$ de $\sim 0.2$. Ficando empatados como segundo modelos mais plausível (ambos com $\triangle \mathrm{AIC}$ menor que 5) os 
modelos para interação biótico-abiótico - com e sem tipo de solo -, que também apresentam $\mathrm{R}^{2}$ próximo a 0.2 .

Por fim, em LDMC, o modelo mais plausível é o de influência biótica com tipo de solo e, em segundo lugar $(\triangle A I C=2.34)$, a influência biótica e abiótica com tipo de solo, com $\mathrm{R}^{2}$ de 0.103 e 0.124 , respectivamente. 


\section{DISCUSSÃO}

Partindo da base da teoria de nicho esperamos que diferenças no ambiente criem situações onde certas características são mais vantajosas que outras e que as diversas espécies respondam a essas diferenças. Esperávamos, portanto, que houvesse correlação entre a estrutura do ambiente e a estrutura dos atributos funcionais. Entretanto, quando tratamos a identidade das espécies recrutadas como variável aleatória, nossos resultados mostram que nenhuma de nossas variáveis ambientais interfere nas variações dos valores dos atributos foliares. Não apenas a teoria prevê que o ambiente esteja relacionado com a estrutura de atributos funcionais, como existe uma diferença qualitativa na estrutura da comunidade entre as áreas de neossolo e espodossolo da parcela permanente, que influenciam de forma evidente tanto a distribuição de espécies e quanto a densidade da vegetação (Lima et al., 2011, Oliveira et al., 2014). Seria razoável esperar que tal diferença ambiental viesse correlacionada com uma mudança na tendência de atributos funcionais, mesmo quando não encontrássemos a influência de dados mais finos de química do solo ou características da vizinhança nos valores de atributos.

No primeiro bloco de análises, de forma geral, os ajustes dos modelos atribuem as variações individuais às variáveis aleatórias (às identidades das espécies) e, ao retirar as espécies como fator na segunda análise, elegemos como modelos mais plausíveis aqueles que incluem tanto variáveis bióticas quanto abióticas. É possível então que a informação mais relevante para prever os valores individuais dos atributos de um indivíduo seja a sua espécie, mas que, na ausência dessa informação, as variáveis ambientais permitem algum grau predição. Em nossa interpretação, os resultados indicam que o ambiente atua na distribuição das espécies e, como a identidade da espécie é um bom preditor para o valor dos atributos, o ambiente acaba atuando como preditor indireto dos valores de atributo quando não temos informação sobre as diferentes espécies, o que é diferente do que originalmente esperávamos: Que o ambiente fosse um preditor dos valores de atributo através das 
estratégia ecológicas dos indivíduos, afetando de forma relativamente homogênea as diferentes espécies.

Quanto à relação entre variáveis bióticas e abióticas, os atributos médios da vizinhança podem ser tão relevantes quanto às variáveis de solo para a montagem da comunidade. É condizente com nossa expectativa teórica que ambas afetem a estrutura da comunidade, e nossa interpretação dos resultados com modelos onde não utilizamos a identidade das espécies como variável aleatória nos leva a crer que este seja o caso, embora a distribuição espacial dos atributos funcionais foliares não seja o melhor indicador desses efeitos.

Ambos os tipos de variáveis, bióticas e abióticas, podem ser usados em conjunto para ganharmos um entendimento mais refinado da comunidade vegetal do que quando utilizamos apenas um dos tipos de variáveis, mas sua relação exata ainda precisa ser explorada. Para melhor entender o mecanismo da interação entre os dois tipos de variáveis, caberia um diferente tipo de análise: Como existem processos pelos quais as variáveis bióticas do ambiente podem ser influenciadas pelas abióticas (pois a estrutura da comunidade pode estar relacionada às características do ambiente), e como algumas das abióticas (como presença de matéria orgânica no solo) podem ser influenciadas por variáveis bióticas, seria adequado uma análise que explore a possível hierarquia ou dependência entre os grupos de variáveis. Esse tipo de análise permitiria, em trabalhos futuros, trazer conclusões mais acuradas e começar a apontar possíveis mecanismos para a relação entre as variáveis bióticas e abióticas, permitindo ao mesmo tempo verificar nossa hipótese de que o ambiente influencia a distribuição espacial dos atributos através da distribuição de espécies.

Contrariando nossas expectativas iniciais, não encontramos uma tendência geral entre fatores ambientais e distribuição espacial dos atributos foliares. Uma possibilidade é de que o gradiente ambiental da parcela não seja, apesar de nossas expectativas, o suficiente para que possamos observar mudanças na estrutura de atributos funcionais em resposta ao ambiente. Entre os fatores que podem afetar a 
variação intraespecífica de atributos funcionais, além de resposta plástica do indivíduo a características do ambiente, há a hereditariedade e o efeito do acaso. É possível que a influência do acaso e hereditariedade nas variações individuais seja maior do que o esperado e que eclipse o efeito das variáveis ambientais. Nesse caso, apesar dos atributos funcionais serem indicadores convenientes das estratégias das plantas de forma ampla, pode ser que a medição indivíduo a indivíduo não traga informações úteis quanto à dinâmica interna de uma comunidade, principalmente se olharmos apenas uma área relativamente pequena, como é o caso da parcela permanente.

Sabemos ainda que as áreas de restinga como um todo trazem sérios filtros ambientais para as espécies de plantas: o solo arenoso, acídico e salino garante que as espécies que encontramos na alta restinga sejam poucas em comparação com a Mata Atlântica de Encosta (Scarano, 2009). É possível que esses filtros ambientais sejam os maiores determinantes na estratégia ecológica das árvores de restinga, ao ponto em que as diferenças ambientais que encontramos dentro da parcela permanente não sejam o bastante para que os indivíduos tenham atributos diferentes em resposta. Além disso, talvez o ambiente seja mais homogêneo do que supomos, pois apesar das raízes normalmente não atingirem grandes profundidades, elas podem se estender horizontalmente ao longo do chão por vários metros. No momento não sabemos dizer ao certo qual pode ser o alcance das raízes na parcela, mas parece razoável esperar que elas se estendam além do sub-quadrado de onde emerge o tronco, ao menos em alguns casos. Nesse caso, do ponto de vista das plantas, o ambiente seria muito mais homogêneo do que esperávamos e uma parte considerável dos padrões de distribuição das espécies são fruto do acaso e da forma como ocorreu a dispersão. Mesmo nesse caso, no entanto, não esperamos que todos os padrões sejam frutos do acaso, apenas que o papel dos processos neutros seja maior do que originalmente suponhamos.

É também possível que cada espécie reaja de forma diferente às mesmas pressões ambientais. Nesse caso, não teríamos uma tendência global, mas várias 
tendências diferentes, uma para cada espécie. Em nossos dados de atributos funcionais dos novos indivíduos, apenas duas espécies possuem mais do que 20 ocorrências, Guatteria australis e Psychotria hastisepala (ambas ocorrendo nos dois tipos de solo). Explorando a possibilidade da variação intraespecífica ser afetada pelo meio, repetimos a análise separadamente para essas duas espécies e encontramos que modelos diferentes são selecionados para cada uma (Tabela 4). Com o devido cuidado ao basear argumentos em um número amostral tão reduzido, seria possível imaginar que variações ambientais dentro da área da parcela não sejam o bastante para criar filtros que favoreçam certas características em detrimento de outras, de tal forma que, embora os atributos dos indivíduos respondam ao ambiente, não observamos tendências gerais nos valores de atributos. Nesse caso, trabalhos focados em explicar o padrão espacial dos atributos funcionais podem ser inconclusivos em áreas de florestas tropicais, onde a riqueza de espécies e a consequente heterogeneidade relacionada às particularidades de cada espécie é muito alta. Trabalhos futuros podem lidar com os obstáculos relacionados à alta riqueza ao utilizar métricas de propriedades emergentes, como a disparidade funcional, ou ao agregar as espécies em grupos que possuem o mesmo tipo de resposta à variações do ambiente, por exemplo através de filogenia ou guildas ecológicas. A primeira abordagem não apenas exige estatísticas mais sofisticadas, mas também um volume de dados maior do que o que dispomos para o trabalho atual, pois não poderia tratar cada indivíduo como uma observação. A segunda abordagem exige agrupar centenas de espécies por sua resposta ao meio, ou assumindo grupos arbitrários com base em expectativas teóricas ou encontrando primeiro os padrões de agrupamento existentes no sistema de estudo, o que poderia em si ser tema de uma nova dissertação.

Em resumo: no primeiro bloco de análises, onde a identidade das espécies foi utilizada como variável aleatória, não encontramos relação entre ambiente e atributos funcionais, indicando que a identidade das espécies seja uma informação mais relevante do que variáveis ambientais. Entretanto, com os resultados da segunda análise, concluímos que tanto variáveis ambientais bióticas quanto abióticas, 
em conjunto, são necessárias para descrever aspectos da estrutura da comunidade, embora os mecanismos pelos quais isso acontece ainda precisem de estudos mais aprofundados. As diferentes respostas das duas análises nos levam à hipótese de que o ambiente seja um preditor indireto para a distribuição de atributos funcionais, através da distribuição de espécies. Também é possível que a importância de processos neutros seja largamente maior do que previamente acreditávamos, que a heterogeneidade ambiental na escala em que trabalhamos não seja tão relevante quanto o esperado ou que diferentes espécies reagem de forma diametralmente diferente às mesmas condições ambientais.

Tabela 4: Os modelos mais plausíveis, selecionados por AIC, para a distribuição espacial de três atributos funcionais de duas espécies da parcela, abaixo do modelo os respectivos valores ajustados de $R^{2}$. "N" representa o número de indivíduos amostrados para cada espécie. No modelo para LDMC de G. australis, foram omitidos dois modelos (C e T) que apresentam $R^{2}=-0.03$; O AIC considera-os igualmente plausíveis ao modelos nulo, pois adicionam explicação aos dados ao incluir apenas uma variável preditora, mas o ajuste desses modelos aos dados é apenas marginalmente superior ao modelo nulo e poderia ser espúria. Da mesma forma, devemos olhar com cautela para os modelos aqui apresentados, pois os mais cheios chegam a ter 18 variáveis.

Modelos mais Plausíveis $(\triangle \mathrm{AIC} \leq 2)$

\begin{tabular}{|c|c|c|c|c|}
\hline \multirow[b]{2}{*}{ Espécie } & \multirow[b]{2}{*}{$\mathbf{N}$} & \\
\hline & & LLA & LSLA & LDMC \\
\hline $\begin{array}{l}\text { Guatteria } \\
\text { australis }\end{array}$ & 35 & $\begin{array}{c}\mathrm{A}+(\mathrm{B}+\mathrm{C})+\mathrm{PC1}:(\mathrm{B}+\mathrm{C})+\mathrm{T} \\
\left(\mathrm{R}^{2}: 0.29\right)\end{array}$ & $\begin{array}{c}A \\
\left(R^{2}: 0.22\right) \\
A+(B+C) \\
\left(R^{2}: 0.23\right) \\
A+T \\
\left(R^{2}: 0.22\right)\end{array}$ & $\begin{array}{c}\text { nulo } \\
\left(R^{2}: 0\right) \\
A+T \\
\left(R^{2}: 0.1\right)\end{array}$ \\
\hline $\begin{array}{l}\text { Psychotria } \\
\text { hastisepala }\end{array}$ & 34 & $\begin{array}{c}\text { nulo } \\
\left(R^{2}: 0\right) \\
(B+C) \\
\left(R^{2}: 0.16\right) \\
(B+C)+T \\
\left(R^{2}: 0.18\right)\end{array}$ & $\begin{array}{c}(B+C) \\
\left(R^{2}: 0.49\right) \\
(B+C)+T \\
\left(R^{2}: 0.48\right)\end{array}$ & $\begin{array}{c}A+(B+C)+P C 1:(B+C) \\
\left(R^{2}: 0.54\right) \\
A+(B+C)+P C 1:(B+C)+T \\
\left(R^{2}: 0.52\right)\end{array}$ \\
\hline
\end{tabular}


Como processos que regem a dinâmica de uma floresta atuam em escalas espaciais e temporais amplas, nós não podemos subestimar a importância do acaso em nossas análises, principalmente quando usamos apenas uma parte dos dados disponíveis. Entretanto, pelo mesmo motivo, não podemos descartar tamanhos pequenos de efeito, já que sua relevância pode ser maior do que aparenta: Um processo que tem pouco efeito aparente sobre milhares de indivíduos, mesmo após décadas, pode gerar padrões relevantes quando atuando em milhões de indivíduos por séculos. A única forma para avançarmos o nosso entendimento na ecologia de comunidades vegetais tropicais é identificando acuradamente os padrões que existem e, para isso, trabalhos que buscam identificar esses padrões precisam ser aperfeiçoados e replicados. 


\section{REFERÊNCIAS}

Adler, P. B., HilleRisLambers, J., \& Levine, J. M. (2007). A niche for neutrality. Ecology Letters, 10(2), 95-104.

Adler, P. B., Smull, D., Beard, K. H., Choi, R. T., Furniss, T., Kulmatiski, A., ... \& Veblen, K. E. (2018). Competition and coexistence in plant communities: intraspecific competition is stronger than interspecific competition. Ecology Letters, 21(9), 1319-1329.

Austin, M. (2007). Species distribution models and ecological theory: a critical assessment and some possible new approaches. Ecological Modelling, 200(1-2), 1-19.

Bonilha, R. M., Casagrande, J. C., Soares, M. R., \& Reis-Duarte, R. M. (2012). Characterization of the soil fertility and root system of restinga forests. Revista Brasileira de Ciência do Solo, 36(6), 1804-1813.

Canham, C. D., Papaik, M. J., Uriarte, M., McWilliams, W. H., Jenkins, J. C., \& Twery, M. J. (2006). Neighborhood analyses of canopy tree competition along environmental gradients in New England forests. Ecological Applications, 16(2), 540-554.

Chapman, H. E. (1966). Diagnostic criteria for plants and soils. Diagnostic Criteria for Plants and Soils.

Condit, R. (1998). Tropical forest census plots: methods and results from Barro Colorado Island, Panama and a comparison with other plots. Springer Science \& Business Media.

Cornelissen, J. H. C., Lavorel, S., Garnier, E., Diaz, S., Buchmann, N., Gurvich, D. E., ... \& Pausas, J. G. (2003). A handbook of protocols for standardised and easy measurement of plant functional traits worldwide. Australian Journal of Botany, 51(4), 335-380.

Gomes, Felipe Haenel, Vidal-Torrado, Pablo, Macías, Felipe, Gherardi, Bruno, \& Perez, Xosé Luiz Otero. (2007). Solos sob vegetação de restinga na Ilha do Cardoso (SP): I - Caracterização e classificação. Revista Brasileira de Ciência do Solo, 31(6), 1563-1580. 
Hubbell, S. P. (2001). The unified neutral theory of biodiversity and biogeography (MPB-32). Princeton University Press.

Hubbell, S. P. (2005). Neutral theory in community ecology and the hypothesis of functional equivalence. Functional Ecology, 19(1), 166-172.

Kraft, N. J., Godoy, O., \& Levine, J. M. (2015). Plant functional traits and the multidimensional nature of species coexistence. Proceedings of the National Academy of Sciences, 112(3), 797-802.

Kraft, N. J., Valencia, R., \& Ackerly, D. D. (2008). Functional traits and niche-based tree community assembly in an Amazonian forest. Science, 322(5901), 580-582.

Keddy, P. A. (1989). Effects of competition from shrubs on herbaceous wetland plants: a 4-year field experiment. Canadian Journal of Botany, 67(3), 708-716.

Keddy, P. A. (1992). Assembly and response rules: two goals for predictive community ecology. Journal of Vegetation Science, 3(2), 157-164.

Kunstler, G., Falster, D., Coomes, D. A., Hui, F., Kooyman, R. M., Laughlin, D. C., ... \& Aiba, M. (2016). Plant functional traits have globally consistent effects on competition. Nature, 529(7585), 204-207.

Levin, S. A. (1970). Community equilibria and stability, and an extension of the competitive exclusion principle. The American Naturalist, 104(939), 413-423.

Lima, R. A. F. D., Oliveira, A. A. D., Martini, A. M. Z., Sampaio, D., Souza, V. C., \& Rodrigues, R. R. (2011). Structure, diversity, and spatial patterns in a permanent plot of a high Restinga forest in Southeastern Brazil. Acta Botanica Brasilica, 25(3), 633-645.

McGill, B. J., Enquist, B. J., Weiher, E., \& Westoby, M. (2006). Rebuilding community ecology from functional traits. Trends in Ecology \& Evolution, 21(4), 178-185. 
Messier, C., Parent, S., \& Bergeron, Y. (1998). Effects of Overstory and Understory Vegetation on the Understory Light Environment in Mixed Boreal Forests. Journal of Vegetation Science, 9(4), 511. doi:10.2307/3237266

Moeslund, J. E., Arge, L., Bøcher, P. K., Dalgaard, T., \& Svenning, J.-C. (2013). Topography as a driver of local terrestrial vascular plant diversity patterns. Nordic Journal of Botany, 31(2), 129-144. doi:10.1111/j.1756-1051.2013.00082.x

Nakagawa, S., Johnson, P.C.D., Schielzeth, H. (2017) The coefficient of determination $\mathrm{R}^{2}$ and intra-class correlation coefficient from generalized linear mixed-effects models revisited and expanded. J. R. Soc. Interface 14: 20170213.

Oliveira, A. A., Vicentini, A., Chave, J., Castanho, C. D. T., Davies, S. J., Martini, A. M., ... \& Souza, V. C. (2014). Habitat specialization and phylogenetic structure of tree species in a coastal Brazilian white-sand forest. Journal of Plant Ecology, 7(2), 134-144.

Ovaskainen, O., Tikhonov, G., Norberg, A., Guillaume Blanchet, F., Duan, L., Dunson, D., ... \& Abrego, N. (2017). How to make more out of community data? A conceptual framework and its implementation as models and software. Ecology Letters, 20(5), 561-576.

Rosindell, J., Hubbell, S. P., He, F., Harmon, L. J., \& Etienne, R. S. (2012). The case for ecological neutral theory. Trends in Ecology \& Evolution, 27(4), 203-208.

Scarano, F. R. (2009). Plant communities at the periphery of the Atlantic rain forest: rare-species bias and its risks for conservation. Biological Conservation, 142(6), 1201-1208.

Schneider, C. A., Rasband, W. S., \& Eliceiri, K. W. (2012). NIH Image to ImageJ: 25 years of image analysis. Nature Methods, 9(7), 671-675.

Temps, R. C., \& Coulson, K. L. (1977). Solar radiation incident upon slopes of different orientations. Solar Energy, 19(2), 179-184. doi:10.1016/0038-092x(77)90056-1 
Uriarte, M., Condit, R., Canham, C. D., \& Hubbell, S. P. (2004). A spatially explicit model of sapling growth in a tropical forest: does the identity of neighbours matter?. Journal of Ecology, 92(2), 348-360.

Uriarte, M., Swenson, N. G., Chazdon, R. L., Comita, L. S., John Kress, W., Erickson, D., ... \& Thompson, J. (2010). Trait similarity, shared ancestry and the structure of neighbourhood interactions in a subtropical wet forest: implications for community assembly. Ecology Letters, 13(12), 1503-1514.

Uriarte, M., Turner, B. L., Thompson, J., \& Zimmerman, J. K. (2015). Linking spatial patterns of leaf litterfall and soil nutrients in a tropical forest: a neighborhood approach. Ecological Applications, 25(7), 2022-2034.

Vellend, M. (2010). Conceptual synthesis in community ecology. The Quarterly Review of Biology, 85(2), 183-206.

Wang, X., Wiegand, T., Kraft, N. J., Swenson, N. G., Davies, S. J., Hao, Z., ... \& Su, S. H. (2016). Stochastic dilution effects weaken deterministic effects of niche-based processes in species rich forests. Ecology, 97(2), 347-360.

Wennekes, P. L.,, J., \& Etienne, R. S. (2012). The neutral-niche debate: a philosophical perspective. Acta Biotheoretica, 60(3), 257-271.

Wiegand, T., Uriarte, M., Kraft, N. J., Shen, G., Wang, X., \& He, F. (2017). Spatially Explicit Metrics of Species Diversity, Functional Diversity, and Phylogenetic Diversity: Insights into Plant Community Assembly Processes. Annual Review of Ecology, Evolution, and Systematics, 48, 329-351. 